\title{
Acid-Stable Oxides for Oxygen Electrocatalysis
}

Wang, Zhenbin; Zheng, Ya-Rong; Chorkendorff, Ib; Norskov, Jens K.

Published in:

ACS Energy Letters

Link to article, DOI:

10.1021/acsenergylett.0c01625

Publication date:

2020

Document Version

Peer reviewed version

Link back to DTU Orbit

Citation (APA):

Wang, Z., Zheng, Y-R., Chorkendorff, I., \& Norskov, J. K. (2020). Acid-Stable Oxides for Oxygen

Electrocatalysis. ACS Energy Letters, 5(9), 2905-2908. https://doi.org/10.1021/acsenergylett.0c01625

\section{General rights}

Copyright and moral rights for the publications made accessible in the public portal are retained by the authors and/or other copyright owners and it is a condition of accessing publications that users recognise and abide by the legal requirements associated with these rights.

- Users may download and print one copy of any publication from the public portal for the purpose of private study or research.

- You may not further distribute the material or use it for any profit-making activity or commercial gain

- You may freely distribute the URL identifying the publication in the public portal

If you believe that this document breaches copyright please contact us providing details, and we will remove access to the work immediately and investigate your claim. 


\title{
Acid-Stable Oxides for Oxygen Electrocatalysis
}

\author{
Zhenbin Wang, ${ }^{\dagger}$ Ya-Rong Zheng, ${ }^{\ddagger}$ Ib Chorkendorff, ${ }^{\ddagger}$ and Jens K. Nørskov ${ }^{*}, \dagger$ \\ $\dagger$ CatTheory, Department of Physics, Technical University of Denmark, 2800 Kongens Lyngby, \\ Denmark
}

$\$$ SurfCat, Department of Physics, Technical University of Denmark, Lyngby, Denmark.

E-mail: jkno@dtu.dk

\begin{abstract}
Assessing materials' stability under operating conditions is of critical importance to the development of device-oriented electrocatalysts, whereas few efforts have been devoted to this on a large scale. In this work, using the Materials Project data, we explore the aqueous stability of 47,814 non-binary metal oxides under typical oxygen reduction/evolution reaction conditions and identify 68 likely acid-stable candidates for oxygen electrocatalysts. We also construct an "acid-stable periodic table" to guide the search of new acid-stable materials for electrocatalysis.
\end{abstract}

Electrocatalysis is fundamental to renewable energy applications by facilitating energy conversion between electricity and chemicals. ${ }^{1}$ The electrochemical process involving oxygen, such as the oxygen reduction reaction (ORR) and the oxygen evolution reaction (OER), however, have become bottlenecks for applications in fuel cells and electrolyzers due to the relatively poor performance of even the best known ORR/OER electrocatalysts. Acid-based devices are more promising in practical applications because of the commercially accessible proton exchange membranes and high current density, ${ }^{2,3}$ but the highly oxidizing and acidic environment severely limits the stability of most materials as catalysts. 
To develop an electrocatalyst for practical applications, we need catalysts that are active and stable. ${ }^{3}$ In the present paper we focus on stability of catalysts under acid condition at potentials relevant to ORR ( $>0.6 \mathrm{~V}$ vs SHE) and OER ( $>1.2 \mathrm{~V}$ vs SHE). First ruling out the unstable catalysts may be the most efficient start of a search procedure. We focus on metal oxide catalysts, given that they are the most likely stable candidates under the oxidizing ORR/OER conditions. Hitherto, transition metal binary oxides have been extensively studied experimentally, ${ }^{4}$ but only very few are promising because of low activity and/or instability under operating conditions. The most famous example is the state-of-the-art OER catalyst, $\mathrm{IrO}_{2}$, which still dissolves at potentials higher than $1.6 \mathrm{~V} .{ }^{5}$ The most extensive search for new oxide electrocatalysts so far is by Shinde et al. ${ }^{6}$ who performed an experimental high-throughput screening on the (Mn-Co-Ta-Sb) $\mathrm{O}_{x}$ chemical space to search for acid-stable OER catalyst.

In this work, building on the extensive works constructing computational Pourbaix diagrams, ${ }^{7-9}$ we evaluated the aqueous stability of 47,814 oxides under typical ORR/OER potentials in strong acid based on the data in the Materials Project. ${ }^{10}$ Based on a set of stability criteria, we identified 68 acid-stable ternary and quaternary oxides. We further constructed an acid-stable periodic table based on a statistical analysis of compositions of these 68 acid-stable oxides and find that oxides containing the elements of $\mathrm{Sb} / \mathrm{Ti} / \mathrm{Sn} / \mathrm{Ge} / \mathrm{Mo} / \mathrm{W}$ tend to have high corrosion resistance in an acidic and oxidative environment.

Figure 1(a) presents the high-throughput screening workflow we developed to identify acidstable materials for ORR/OER. We began our search among the 47,814 oxides included in the Materials Project database. ${ }^{10}$ The aqueous stability of these materials is then computed in the potential range of $0.6-1.0 \mathrm{~V}$ (vs. SHE) and $1.2-2.0 \mathrm{~V}$ (vs. SHE) for ORR and OER, respectively, at $\mathrm{pH}=0$. We note that, in the literature the high-throughput screenings ${ }^{11-13}$ based on aqueous stability often only evaluate the materials' stability at a given potential, which may be insufficient to identify a "truly" stable candidate, since in experiment the catalyst is often cycled over a range of operating potentials at certain $\mathrm{pH}$. Previous benchmark efforts by Singh et al. ${ }^{8}$ have shown that the aqueous stability can be assessed by computing the material's Gibbs free energy difference 
$\left(\Delta G_{\mathrm{pbx}}\right)$ with respect to the stable domains on the Pourbaix diagram as a function of $\mathrm{pH}$ and potential, as illustrated in Figure 1(b). In principle, a stable material should have $\Delta G_{\mathrm{pbx}}=0$, but it was suggested that a threshold of $\Delta G_{\mathrm{pbx}}$ of $0.5 \mathrm{eV} /$ atom yields materials with a reasonable stability against corrosion due to self-passivation or the formation of a more stable solid-state phase. ${ }^{8}$ We therefore adopt this criterion in our screening. In addition, we computed the band gap $\left(E_{\mathrm{g}}\right)$ of the most promising materials using the screened hybrid Heyd-Scuseria-Ernzerhof (HSE) functional $^{14}$ in order to evaluate their electronic properties. We also calculated the energy above the convex hull $\left(E_{\text {hull }}\right)$, as depicted in Figure 1(c), for these candidates to estimate their phase stability. $E_{\text {hull }}$ measures the energy involved in the decomposition reaction from the studied material to the most stable phase in the solid-state phase diagram. The phase diagram was constructed using the energies of relevant compounds obtained from the Materials Project. ${ }^{10,15,16}$

By applying the aqueous stability criterion of $\Delta G_{\mathrm{pbx}}<0.5 \mathrm{eV} /$ atom on the 47,814 oxides, we obtained 68 non-binary acid-stable oxides, shown in Table 1 and Table S1. (Table was separated based on the band gap of $1.0 \mathrm{eV}$ due to page limit.) This considerable reduction of potential candidates highlights the importance of aqueous stability evaluation when searching an electrocatalyst for practical application. In Table 1 and Table $\mathrm{S} 1$, the smaller $\Delta G_{\mathrm{pbx}}^{\mathrm{X}}(\mathrm{X}=\mathrm{ORR} / \mathrm{OER})$, the more stable compounds against aqueous decomposition. We include $E_{\mathrm{g}}$ in the tables to provide an indication of the electronic conductivity, metals $\left(E_{\mathrm{g}}=0\right)$ having the best electronic conductivity and the large band gap oxides the lowest. The small gap, semiconducting materials may have a certain electronic conductivity through defects and impurities. The $E_{\text {hull }}$ is added in the tables as a proxy of material's synthesizability. A stable phase by definition yields $E_{\text {hull }}=0$ and the higher the $E_{\text {hull, }}$, the more unstable this material is and, consequently, the more challenging it might be to synthesize it.

Four of these 68 materials, $\mathrm{M}\left(\mathrm{SbO}_{3}\right)_{2}(\mathrm{M}=\mathrm{Mn} / \mathrm{Fe} / \mathrm{Co} / \mathrm{Ni})$ have been investigated in experiment as electrocatalysts for OER and the chlorine evolution reaction (CER). ${ }^{17,18}$ It was found in experiment that $\mathrm{M}\left(\mathrm{SbO}_{3}\right)_{2}(\mathrm{M}=\mathrm{Fe} / \mathrm{Co} / \mathrm{Ni})$ showed high corrosion resistance under OER and CER operating conditions, which is consistent with our predictions. (Table 1 and Table S1) Close exam- 
ination of the aqueous decomposition products of these three acid-stable compounds reveals that the formation of more thermodynamically acid-stable solid phases is mainly responsible for this high corrosion resistance. For example, the formation of $\mathrm{FeSbO}_{4}$ and antimony oxides $\left(\mathrm{Sb}_{2} \mathrm{O}_{5}\right.$ or $\left.\mathrm{Sb}_{3} \mathrm{O}_{8}\right)$ for $\mathrm{Fe}\left(\mathrm{SbO}_{3}\right)_{2}$ under potentials of $1.2-2.0 \mathrm{~V}$ at $\mathrm{pH}=0$, shown in Figure 2(a), prevents its aqueous decomposition because of a higher solid-solid phase transition barrier $(1 \mathrm{eV}) .{ }^{8} \mathrm{Mn}\left(\mathrm{SbO}_{3}\right)_{2}$ was experimentally observed to exhibit a high dissolution rate at potentials higher than $1.8 \mathrm{~V}$ (vs. RHE), which can be attributed to the large Pourbaix decomposition free energy $\left(\Delta G_{\mathrm{pbx}}\right)$, shown in Figure 2(b).

On a side note, we found that some materials (e.g. $\left.\mathrm{Sn}\left(\mathrm{WO}_{3}\right)_{18}\right)$ are particularly promising as supporting materials as alternatives to carbon-support in $\mathrm{Pt} / \mathrm{C}$ or for single-atom electrocatalysis because of its wide aqueous stability window and small $E_{\mathrm{g}}$. Generally, $3 d$ transition metal elements (e.g. $\mathrm{Mn} / \mathrm{Fe} / \mathrm{Co} / \mathrm{Ni}$ ) in compounds serve as the catalytic center for chemical reaction, and we note that the majority compounds identified as possible support materials do not contain these elements, signifying that they are unlikely to be active for ORR/OER by themselves.

As an aid to future design efforts for acid-stable electrocatalyst, we built an "acid-stable period table" reflecting the composition of the 68 stable oxides, shown in Figure 3. We observe that $\mathrm{Sb} / \mathrm{Ti} / \mathrm{Sn} / \mathrm{Ge} / \mathrm{Mo} / \mathrm{W}$-based oxides have a preference of being stable in strong acid. This insight is of great benefit to search for new stable and active electrocatalyst for ORR and OER using combinatorial chemistry screening or machine learning. ${ }^{6,19,20}$

\section{Acknowledgement}

This work was supported by Toyota Research Institute.

\section{Supporting Information Available}

The Supporting Information is available free of charge on the ACS Publications website at DOI: XXXX 
Methods on density functional theory calculations and aqueous stability analysis; Table S1 summarizing the calculated properties of the rest 53 acid-stable oxides

\section{References}

(1) She, Z. W.; Kibsgaard, J.; Dickens, C. F.; Chorkendorff, I.; Nørskov, J. K.; Jaramillo, T. F. Combining theory and experiment in electrocatalysis: Insights into materials design. Science 2017, 355, eaad4998.

(2) Yang, S.; Verdaguer-Casadevall, A.; Arnarson, L.; Silvioli, L.; Čolić, V.; Frydendal, R.; Rossmeisl, J.; Chorkendorff, I.; Stephens, I. E. Toward the Decentralized Electrochemical Production of H2O2: A Focus on the Catalysis. ACS Catalysis 2018, 8, 4064-4081.

(3) Kibsgaard, J.; Chorkendorff, I. Considerations for the scaling-up of water splitting catalysts. Nature Energy 2019, 4, 430-433.

(4) Liu, M.; Wang, L.; Zhao, K.; Shi, S.; Shao, Q.; Zhang, L.; Sun, X.; Zhao, Y.; Zhang, J. Atomically dispersed metal catalysts for the oxygen reduction reaction: synthesis, characterization, reaction mechanisms and electrochemical energy applications. Energy \& Environmental Science 2019, 2890-2923.

(5) Kasian, O.; Grote, J.-P.; Geiger, S.; Cherevko, S.; Mayrhofer, K. J. J. The Common Intermediates of Oxygen Evolution and Dissolution Reactions during Water Electrolysis on Iridium. Angewandte Chemie International Edition 2018, 57, 2488-2491.

(6) Shinde, A.; Jones, R. J.; Guevarra, D.; Mitrovic, S.; Becerra-Stasiewicz, N.; Haber, J. A.; Jin, J.; Gregoire, J. M. High-Throughput Screening for Acid-Stable Oxygen Evolution Electrocatalysts in the (Mn-Co-Ta-Sb)Ox Composition Space. Electrocatalysis 2015, 6, 229236. 
(7) Persson, K. A.; Waldwick, B.; Lazic, P.; Ceder, G. Prediction of solid-aqueous equilibria: Scheme to combine first-principles calculations of solids with experimental aqueous states. Physical Review B 2012, 85, 235438.

(8) Singh, A. K.; Zhou, L.; Shinde, A.; Suram, S. K.; Montoya, J. H.; Winston, D.; Gregoire, J. M.; Persson, K. A. Electrochemical Stability of Metastable Materials. Chemistry of Materials 2017, 29, 10159-10167.

(9) Patel, A.; Nørskov, J. K.; Persson, K. A.; Montoya, J. H. Efficient Pourbaix diagrams of many-element compounds. 2019, 1-11.

(10) Jain, A.; Ong, S. P.; Hautier, G.; Chen, W.; Richards, W. D.; Dacek, S.; Cholia, S.; Gunter, D.; Skinner, D.; Ceder, G.; Persson, K. A. Commentary: The materials project: A materials genome approach to accelerating materials innovation. APL Materials 2013, 1, 011002.

(11) Jain, A.; Wang, Z.; Nørskov, J. K. Stable Two-Dimensional Materials for Oxygen Reduction and Oxygen Evolution Reactions. ACS Energy Letters 2019, 4, 1410-1411.

(12) Singh, A. K.; Montoya, J. H.; Gregoire, J. M.; Persson, K. A. Robust and synthesizable photocatalysts for $\mathrm{CO} 2$ reduction: a data-driven materials discovery. Nature Communications 2019, $10,443$.

(13) Back, S.; Tran, K.; Ulissi, Z. W. Discovery of Acid-Stable Oxygen Evolution Catalysts : High-throughput Computational Screening of Equimolar Bimetallic Oxides. ACS Applied Materials \& Interfaces 2020, Just Accepted Manuscript.

(14) Heyd, J.; Scuseria, G. E.; Ernzerhof, M. Hybrid functionals based on a screened Coulomb potential. Journal of Chemical Physics 2003, 118, 8207-8215.

(15) Ong, S. P.; Richards, W. D.; Jain, A.; Hautier, G.; Kocher, M.; Cholia, S.; Gunter, D.; Chevrier, V. L.; Persson, K. A.; Ceder, G. Python Materials Genomics (pymatgen): A ro- 
bust, open-source python library for materials analysis. Computational Materials Science 2013, 68, 314-319.

(16) Ong, S. P.; Cholia, S.; Jain, A.; Brafman, M.; Gunter, D.; Ceder, G.; Persson, K. A. The Materials Application Programming Interface (API): A simple, flexible and efficient API for materials data based on REpresentational State Transfer (REST) principles. Computational Materials Science 2015, 97, 209-215.

(17) Moreno-Hernandez, I. A.; MacFarland, C. A.; Read, C. G.; Papadantonakis, K. M.; Brunschwig, B. S.; Lewis, N. S. Crystalline nickel manganese antimonate as a stable wateroxidation catalyst in aqueous 1.0 M H 2 SO 4. Energy \& Environmental Science 2017, 10, 2103-2108.

(18) Moreno-Hernandez, I. A.; Brunschwig, B. S.; Lewis, N. S. Crystalline nickel, cobalt, and manganese antimonates as electrocatalysts for the chlorine evolution reaction. Energy \& Environmental Science 2019, 12, 1241-1248.

(19) Wang, Z.; Ha, J.; Kim, Y. H.; Im, W. B.; McKittrick, J.; Ong, S. P. Mining Unexplored Chemistries for Phosphors for High-Color-Quality White-Light-Emitting Diodes. Joule 2018, 2, 914-926.

(20) Ye, W.; Chen, C.; Wang, Z.; Chu, I.-H.; Ong, S. P. Deep neural networks for accurate predictions of crystal stability. Nature Communications 2018, 9, 3800. 
Table 1: Calculated properties of 15 acid-stable oxides. $\Delta G_{\mathrm{pbx}}^{\mathrm{X}}(\mathrm{X}=\mathrm{ORR} / \mathrm{OER})(\mathrm{eV} / \mathrm{atom})$ is the maximum Pourbaix decomposition free energy in the potential range of interest. Band gap (eV) was calculated using the HSE functional. MP-ID is material id in the Materials Project database. Materials are sorted by their phase stability, $E_{\text {hull }}$ (meV/atom).

\begin{tabular}{|c|c|c|c|c|c|c|}
\hline Materials & Space group & $E_{\text {hull }}$ & $\Delta G_{\mathrm{pbx}}^{\mathrm{ORR}} / \Delta G_{\mathrm{pbx}}^{\mathrm{OER}}$ & Aqueous decomposition products & Band gap & MP-ID \\
\hline $\mathrm{Fe}\left(\mathrm{SbO}_{3}\right)_{2}$ & $\mathrm{P} 4_{2} / \mathrm{mnm}$ & 0 & $0 / 0.328$ & $\begin{array}{c}\mathrm{Fe}\left(\mathrm{SbO}_{3}\right)_{2}(\mathrm{~s})(\mathrm{E}=0.6-1.0 \mathrm{~V}) \\
\mathrm{Sb}_{2} \mathrm{O}_{5}(\mathrm{~s})+\mathrm{FeSbO}_{4}(\mathrm{~s})(\mathrm{E}=1.2-1.95 \mathrm{~V}) \\
\mathrm{FeSbO}_{4}(\mathrm{~s})+\mathrm{Sb}_{3} \mathrm{O}_{8}(\mathrm{~s})(\mathrm{E}=1.96-2.0 \mathrm{~V})\end{array}$ & 0 & mp-541194 \\
\hline $\mathrm{Co}\left(\mathrm{SbO}_{3}\right)_{2}$ & $\mathrm{P} 4_{2} / \mathrm{mnm}$ & 0 & $0.005 / 0.154$ & $\begin{array}{c}\mathrm{Co}^{2+}+\mathrm{SbO}^{+}(\mathrm{E}=0.6 \mathrm{~V}) \\
\mathrm{Co}\left(\mathrm{SbO}_{3}\right)_{2}(\mathrm{~s})(\mathrm{E}=0.61-1.0,1.2-1.78 \mathrm{~V}) \\
\mathrm{CoO}_{2}(\mathrm{~s})+\mathrm{Sb}_{2} \mathrm{O}_{5}(\mathrm{~s})(\mathrm{E}=1.79-1.95 \mathrm{~V}) \\
\mathrm{CoO}_{2}(\mathrm{~s})+\mathrm{Sb}_{3} \mathrm{O}_{8}(\mathrm{~s})(\mathrm{E}=1.96-2.0 \mathrm{~V})\end{array}$ & 0.92 & mp-24845 \\
\hline $\mathrm{Mn}\left(\mathrm{SbO}_{3}\right)_{2}$ & P321 & 0 & $0.078 /-$ & $\begin{array}{c}\mathrm{SbO}^{+}+\mathrm{Mn}^{2+}(\mathrm{E}=0.6-0.62 \mathrm{~V}) \\
\mathrm{SbO}_{2}(\mathrm{~s})+\mathrm{Mn}^{2+}(\mathrm{E}=0.63-0.69 \mathrm{~V}) \\
\mathrm{Mn}\left(\mathrm{SbO}_{3}\right)_{2}(\mathrm{~s})(\mathrm{E}=0.7-1.0 \mathrm{~V})\end{array}$ & 0.78 & $\mathrm{mp}-25043$ \\
\hline $\mathrm{Mn}\left(\mathrm{SbO}_{3}\right)_{2}$ & $\mathrm{P} 4_{2} / \mathrm{mnm}$ & 1 & $0.124 /-$ & $\begin{array}{c}\mathrm{SbO}^{+}+\mathrm{Mn}^{2+}(\mathrm{E}=0.6-0.62 \mathrm{~V}) \\
\mathrm{Mn}^{2+}+\mathrm{SbO}_{2}(\mathrm{~s})(\mathrm{E}=0.63-0.69 \mathrm{~V}) \\
\mathrm{Mn}\left(\mathrm{SbO}_{3}\right)_{2}(\mathrm{~s})(\mathrm{E}=0.7-1.0 \mathrm{~V})\end{array}$ & 0 & mp-763546 \\
\hline $\mathrm{Sn}\left(\mathrm{WO}_{3}\right)_{18}$ & Pmmn & 13 & $0.247 / 0.458$ & $\mathrm{WO}_{3}(\mathrm{~s})+\mathrm{SnO}_{2}(\mathrm{~s})(\mathrm{E}=0.6-1.0,1.2-2.0 \mathrm{~V})$ & 0 & mp-705699 \\
\hline $\mathrm{Sb}_{2} \mathrm{Mo}_{10} \mathrm{O}_{31}$ & Pma2 & 17 & $0.311 /-$ & $\begin{array}{c}\mathrm{Mo}_{8} \mathrm{O}_{23}(\mathrm{~s})+\mathrm{SbO}^{+}(\mathrm{E}=0.6-0.61 \mathrm{~V}) \\
\mathrm{SbO}^{+}+\mathrm{MoO}_{3}(\mathrm{~s})(\mathrm{E}=0.62 \mathrm{~V}) \\
\mathrm{MoO}_{3}(\mathrm{~s})+\mathrm{SbO}_{2}(\mathrm{~s})(\mathrm{E}=0.63-0.77 \mathrm{~V}) \\
\mathrm{Sb}_{2} \mathrm{O}_{5}(\mathrm{~s})+\mathrm{MoO}_{3}(\mathrm{~s})(\mathrm{E}=0.78-1.0 \mathrm{~V})\end{array}$ & 0 & mp-609510 \\
\hline $\mathrm{MoWO}_{6}$ & $\mathrm{P} 2{ }_{1} / \mathrm{c}$ & 36 & $0.152 / 0.150$ & $\begin{array}{c}\mathrm{WO}_{3}(\mathrm{~s})+\mathrm{Mo}_{8} \mathrm{O}_{23}(\mathrm{~s})(\mathrm{E}=0.6-0.61 \mathrm{~V}) \\
\mathrm{WO}_{3}(\mathrm{~s})+\mathrm{MoO}_{3}(\mathrm{~s})(\mathrm{E}=0.62-1.0,1.2-2.0 \mathrm{~V})\end{array}$ & 0.73 & mvc-5033 \\
\hline $\mathrm{Mn}\left(\mathrm{SbO}_{3}\right)_{2}$ & $\mathrm{C} 2 / \mathrm{m}$ & 43 & $0.250 /-$ & $\begin{array}{c}\mathrm{SbO}^{+}+\mathrm{Mn}^{2+}(\mathrm{E}=0.6-0.62 \mathrm{~V}) \\
\mathrm{SbO}_{2}(\mathrm{~s})+\mathrm{Mn}^{2+}(\mathrm{E}=0.63-0.69 \mathrm{~V}) \\
\mathrm{Mn}\left(\mathrm{SbO}_{3}\right)_{2}(\mathrm{~s})(\mathrm{E}=0.7-1.0 \mathrm{~V})\end{array}$ & 0 & mp-690561 \\
\hline $\mathrm{Ge}_{2} \mathrm{MoO}_{6}$ & $\mathrm{C} 2 / \mathrm{c}$ & 62 & $0.477 /-$ & $\begin{array}{c}\mathrm{Mo}_{8} \mathrm{O}_{23}(\mathrm{~s})+\mathrm{GeO}_{2}(\mathrm{~s})(\mathrm{E}=0.6-0.61 \mathrm{~V}) \\
\mathrm{GeO}_{2}(\mathrm{~s})+\mathrm{MoO}_{3}(\mathrm{~s})(\mathrm{E}=0.62-1.0 \mathrm{~V})\end{array}$ & 1.00 & mp-1043238 \\
\hline $\mathrm{Fe}\left(\mathrm{SbO}_{3}\right)_{4}$ & P1 & 82 & $-/ 0.317$ & $\begin{array}{l}\mathrm{Sb}_{2} \mathrm{O}_{5}(\mathrm{~s})+\mathrm{FeSbO}_{4}(\mathrm{~s})(\mathrm{E}=1.2-1.95 \mathrm{~V}) \\
\mathrm{FeSbO}_{4}(\mathrm{~s})+\mathrm{Sb}_{3} \mathrm{O}_{8}(\mathrm{~s})(\mathrm{E}=1.96-2.0 \mathrm{~V})\end{array}$ & 0 & mp-770991 \\
\hline $\mathrm{SnSbO}_{4}$ & $\mathrm{Cmmm}$ & 90 & $0.386 /-$ & $\begin{array}{c}\mathrm{SnO}_{2}(\mathrm{~s})+\mathrm{SbO}^{+}(\mathrm{E}=0.6-0.62 \mathrm{~V}) \\
\mathrm{SbO}_{2}(\mathrm{~s})+\mathrm{SnO}_{2}(\mathrm{~s})(\mathrm{E}=0.63-0.77 \mathrm{~V}) \\
\mathrm{Sb}_{2} \mathrm{O}_{5}(\mathrm{~s})+\mathrm{SnO}_{2}(\mathrm{~s})(\mathrm{E}=0.78-1.0 \mathrm{~V})\end{array}$ & 0 & mp-1218921 \\
\hline $\mathrm{CoSbO}_{4}$ & Imma & 92 & $-/ 0.477$ & $\begin{array}{c}\mathrm{Co}\left(\mathrm{SbO}_{3}\right)_{2}(\mathrm{~s})+\mathrm{Co}^{2+}(\mathrm{E}=1.2-1.59 \mathrm{~V}) \\
\mathrm{Co}\left(\mathrm{SbO}_{3}\right)_{2}(\mathrm{~s})+\mathrm{CoO}_{2}(\mathrm{~s})(\mathrm{E}=1.6-1.78 \mathrm{~V}) \\
\mathrm{Sb}_{2} \mathrm{O}_{5}(\mathrm{~s})+\mathrm{CoO}_{2}(\mathrm{~s})(\mathrm{E}=1.79-1.95 \mathrm{~V}) \\
\mathrm{CoO}_{2}(\mathrm{~s})+\mathrm{Sb}_{3} \mathrm{O}_{8}(\mathrm{~s})(\mathrm{E}=1.96-2.0 \mathrm{~V})\end{array}$ & 0.94 & mp-765886 \\
\hline $\mathrm{Hg}\left(\mathrm{SbO}_{3}\right)_{2}$ & $\mathrm{P} 4_{2} / \mathrm{mnm}$ & 96 & $0.289 / 0.289$ & $\mathrm{Hg}\left(\mathrm{SbO}_{3}\right)_{2}(\mathrm{~s})(\mathrm{E}=0.6-1.0,1.2-2.0 \mathrm{~V})$ & 0 & mp-768291 \\
\hline $\mathrm{Ge}_{2} \mathrm{SbO}_{6}$ & $\mathrm{C} 2 / \mathrm{c}$ & 99 & $0.373 /-$ & $\begin{array}{c}\mathrm{SbO}^{+}+\mathrm{GeO}_{2}(\mathrm{~s})(\mathrm{E}=0.6-0.62 \mathrm{~V}) \\
\mathrm{GeO}_{2}(\mathrm{~s})+\mathrm{SbO}_{2}(\mathrm{~s})(\mathrm{E}=0.63-0.77 \mathrm{~V}) \\
\mathrm{Sb}_{2} \mathrm{O}_{5}(\mathrm{~s})+\mathrm{GeO}_{2}(\mathrm{~s})(\mathrm{E}=0.78-1.0 \mathrm{~V})\end{array}$ & 0 & mp-1042897 \\
\hline $\mathrm{Ge}_{2} \mathrm{SbO}_{6}$ & $\mathrm{P} 2{ }_{1} / \mathrm{c}$ & 114 & $0.420 /-$ & $\begin{array}{c}\mathrm{GeO}_{2}(\mathrm{~s})+\mathrm{SbO}^{+}(\mathrm{E}=0.6-0.62 \mathrm{~V}) \\
\mathrm{SbO}_{2}(\mathrm{~s})+\mathrm{GeO}_{2}(\mathrm{~s})(\mathrm{E}=0.63-0.77 \mathrm{~V}) \\
\mathrm{Sb}_{2} \mathrm{O}_{5}(\mathrm{~s})+\mathrm{GeO}_{2}(\mathrm{~s})(\mathrm{E}=0.78-1.0 \mathrm{~V})\end{array}$ & 0 & mp-1043019 \\
\hline
\end{tabular}




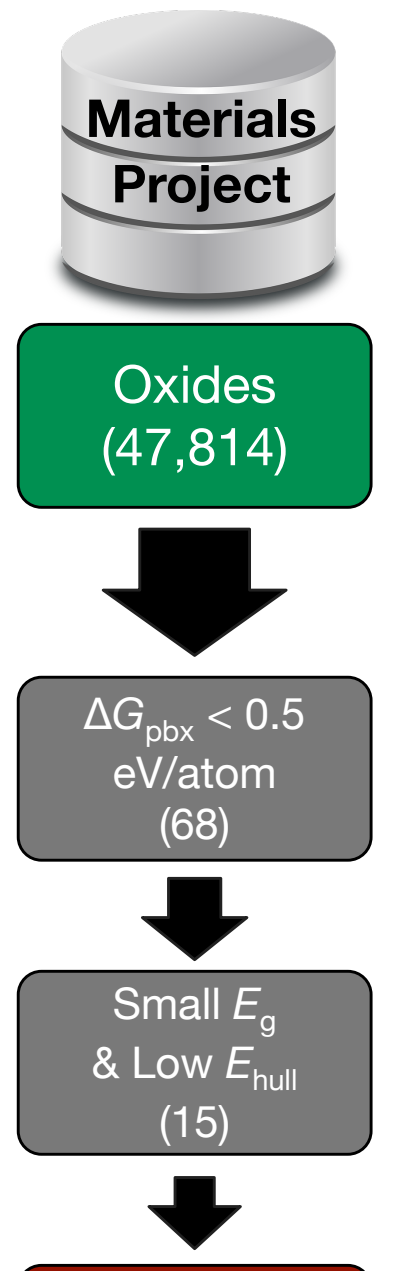

Promising candidates

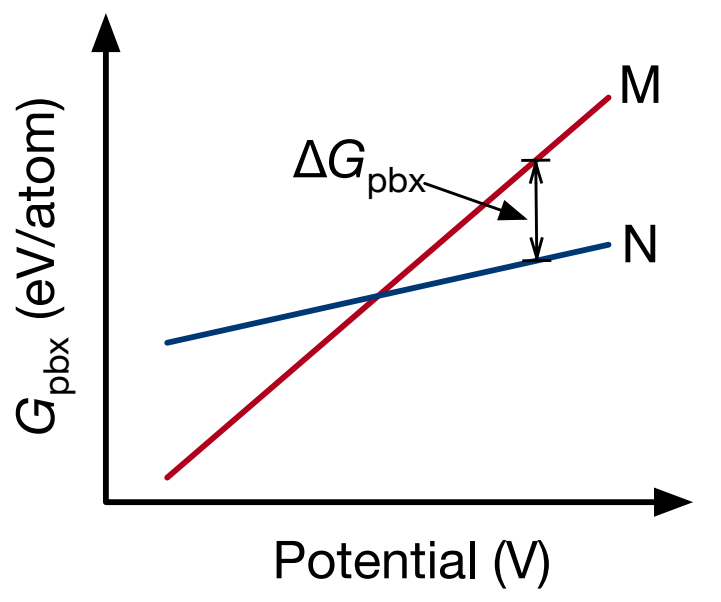

(b)

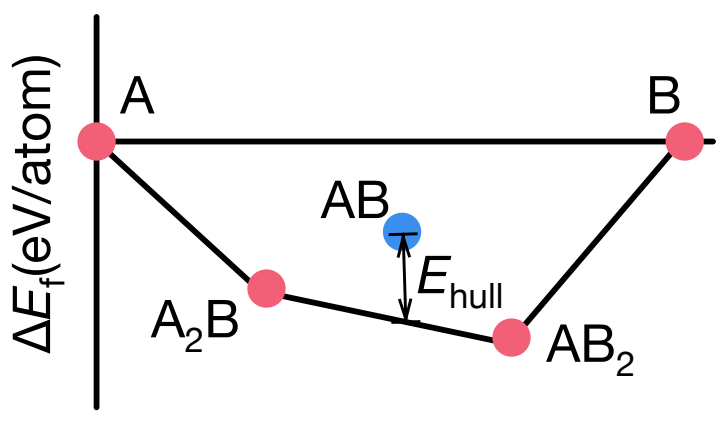

(c)

(a)

Figure 1: (a) Computational high-throughput screening workflow for identifying acid-stable oxides for electrocatalysis. Number in parenthesis is the amount of identified candidates. (b) Schematics of the Pourbaix decomposition free energy $\left(\Delta G_{\mathrm{pbx}}\right)$ as a functional of potential at certain $\mathrm{pH}$, where $\mathrm{M}$ and $\mathrm{N}$ denote two distinct compounds. (c) Illustration of the energy above hull ( $\left.E_{\text {hull }}\right)$ for an A-B system, where pink and blue solid circles are stable and unstable phases, respectively. The line connecting the stables phases is the convex hull. 
(a)

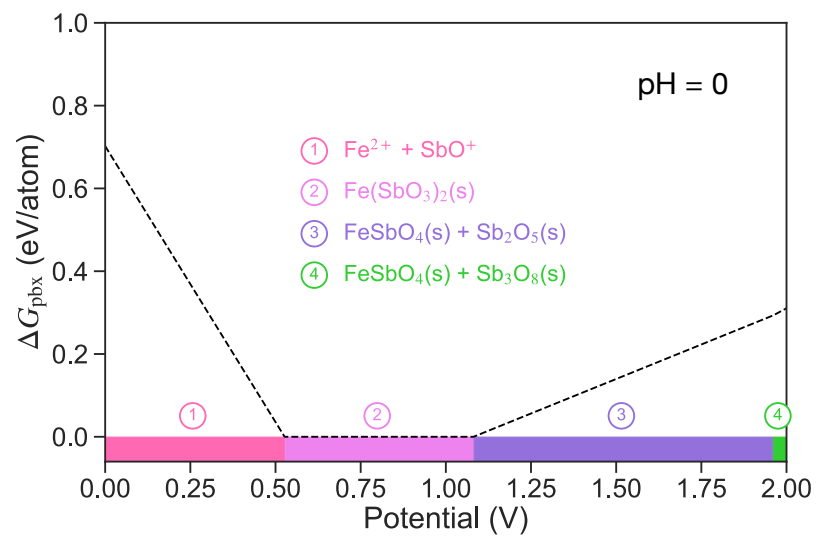

(b)

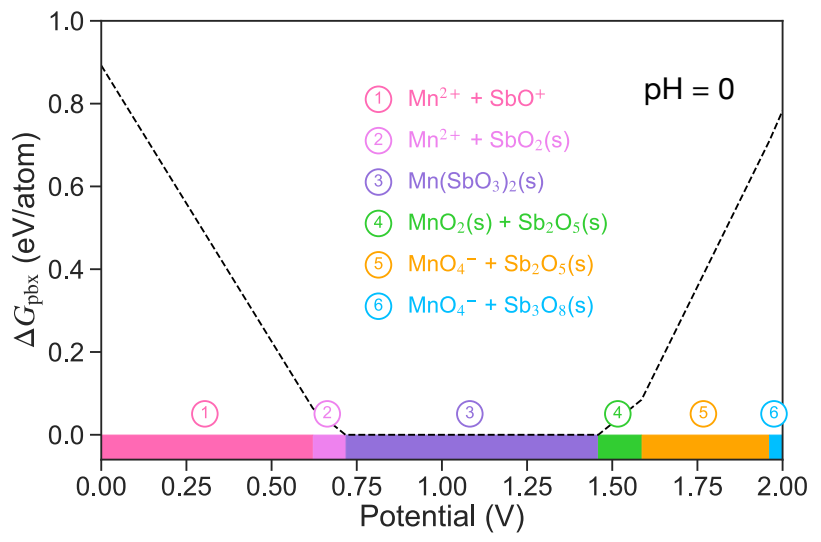

Figure 2: Calculated Pourbaix decomposition free energy $\Delta G_{\mathrm{pbx}}$ of (a) $\mathrm{Fe}\left(\mathrm{SbO}_{3}\right)_{2}$ and (b) $\mathrm{Mn}\left(\mathrm{SbO}_{3}\right)_{2}$ from the potential $0-2.0 \mathrm{~V}$ (vs. $\mathrm{SHE}$ ) at $\mathrm{pH}=0$. The projection of $\Delta G_{\mathrm{pbx}}$ onto the potential axis highlights the stable species at the corresponding regions. 


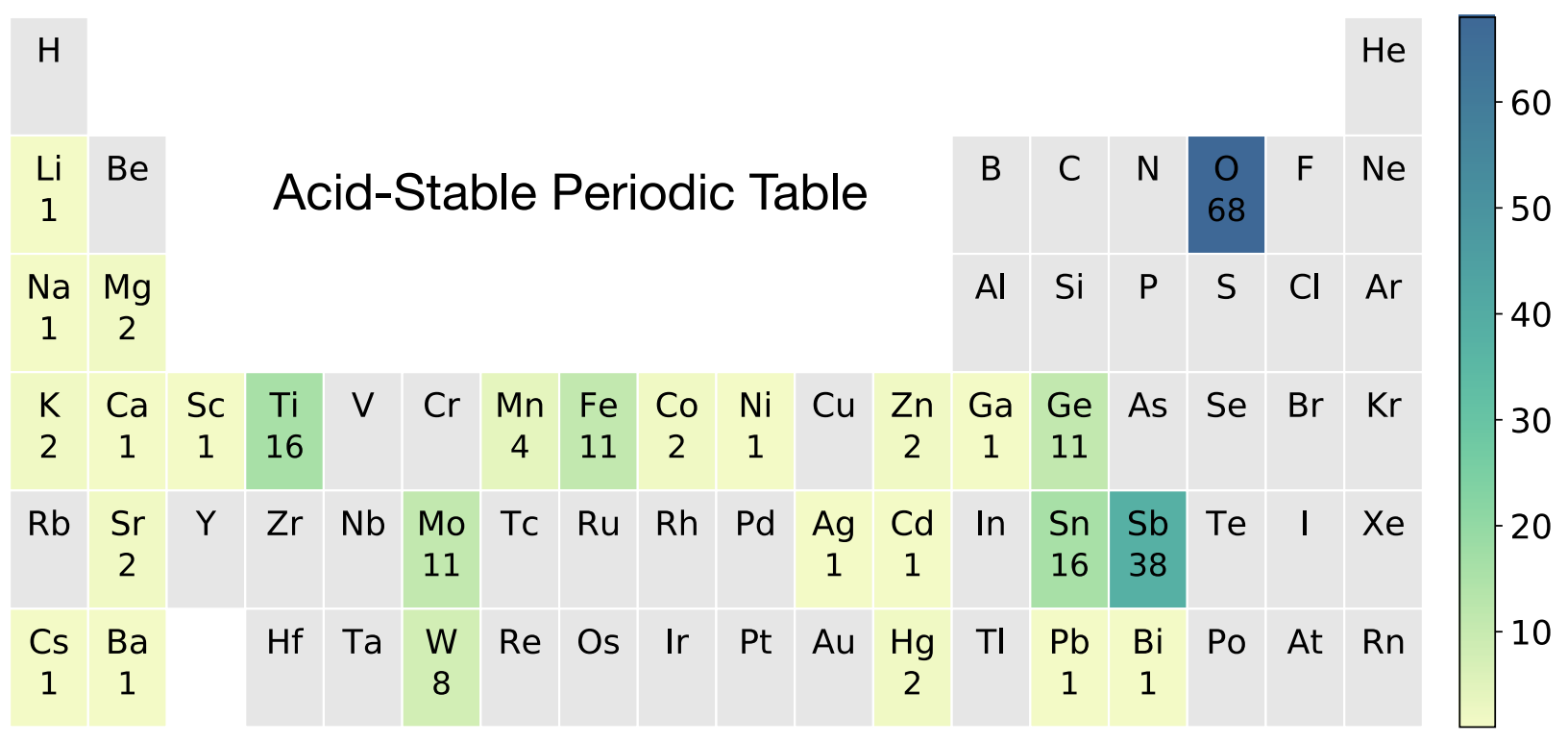

Figure 3: Frequency at which each element consists in acid-stable oxides. Elements with zero frequency are shaded in gray. Lanthanoids and actinoids are omitted for clarity since no oxide containing these elements was predicted to be stable. 


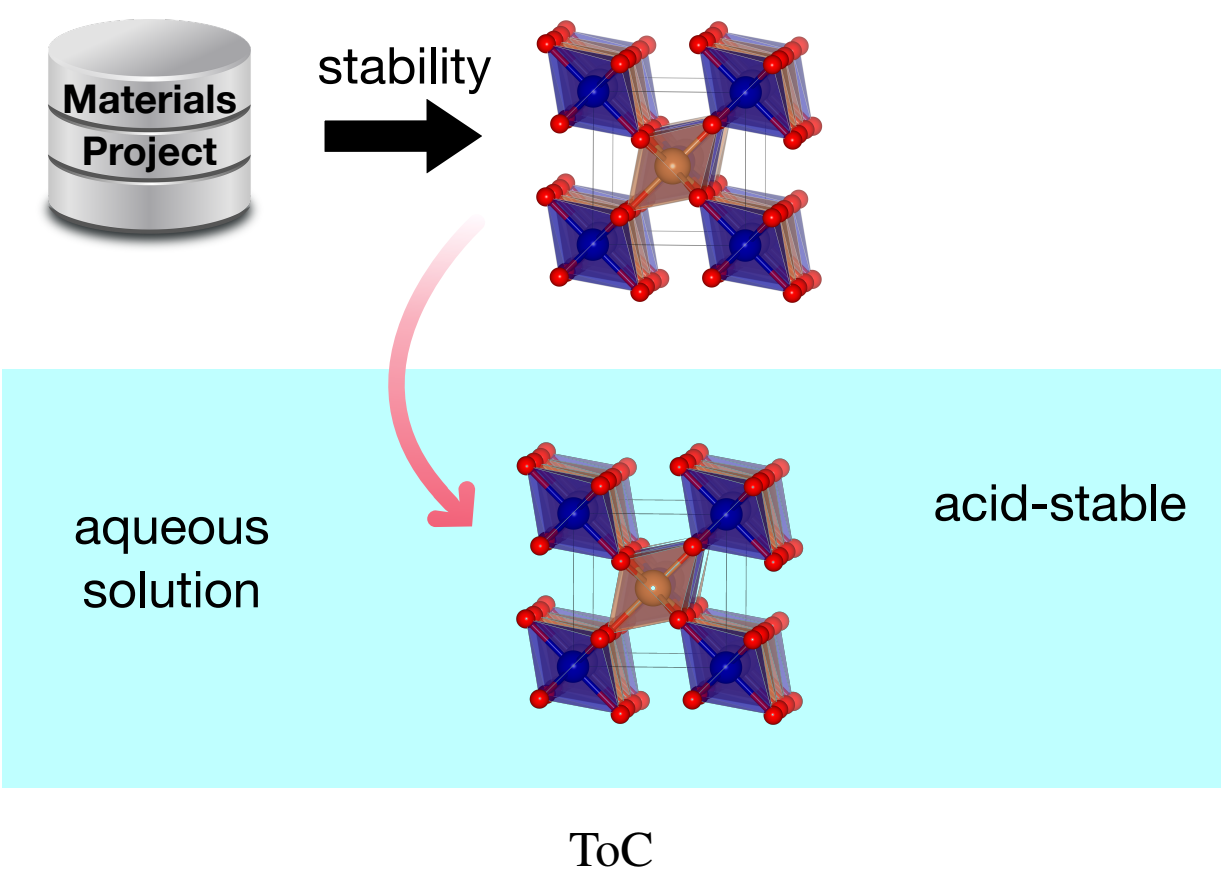




\section{SUPPORTING INFORMATION}

\section{Acid-Stable Oxides for Oxygen Electrocatalysis}

Zhenbin Wang, ${ }^{\dagger}$ Ya-Rong Zheng, ${ }^{\ddagger}$ lb Chorkendorff, ${ }^{\ddagger}$ and Jens Kehlet Nørskov*, ${ }^{*}$

$\dagger$ CatTheory, Department of Physics, Technical University of Denmark, 2800 Kongens Lyngby, Denmark

$\ddagger$ SurfCat, Department of Physics, Technical University of Denmark, Lyngby, Denmark.

E-mail: jkno@dtu.dk 


\section{Computational Method}

\section{Density functional theory calculation}

The density functional theory calculations were performed using the Vienna ab initio simulation package (VASP) within the projector-augmented wave method. ${ }^{1,2}$ The band gap of 68 identified acid-stable oxides were calculated using the screened hybrid Heyd-Scuseria-Ernzerhof (HSE) ${ }^{3,4}$ functional, with a plane wave energy cutoff of $520 \mathrm{eV}$ and a $k$-point density at least of 50 per $\AA^{-3}$. All other energy relevant properties were obtained from the Materials Project via the Materials Project REST API. ${ }^{5,6}$ All data analysis were carried out using the Python Materials Genomics (pymatgen) package. ${ }^{7}$

\section{Aqueous stability analysis}

The aqueous stability was evaluated by computing Pourbaix diagram of each material using the Materials Project methods. ${ }^{8-10}$ Here, we briefly summarize the basic ideas and details are directed to the cited references. In the Pourbaix diagram, the stable domains are determined based on the knowledge of all possible equilibrium redox reactions in the chemical composition of interest. The redox reaction for a given material in an aqueous medium is described by:

$$
a[\text { Reactants }]+\mathrm{bH}_{2} \mathrm{O} \Leftrightarrow \mathrm{c}[\text { Products }]+\mathrm{dH}^{+}+\mathrm{ne}^{-}
$$

The Gibbs free energy change $\left(\Delta G_{\mathrm{rxn}}\right)$ of this reaction can be computed by the free energy change of the reaction under standard conditions. At equilibrium, the Nernst equation,

$$
-n F E^{\prime}=\Delta G_{\mathrm{rxn}}=\Delta G_{\mathrm{rxn}}^{\mathrm{o}}+R T \ln \mathrm{Q}=\Delta G_{\mathrm{rxn}}^{\mathrm{o}}+R T \ln \frac{\left(\mathrm{a}_{\mathrm{P}}\right)^{\mathrm{c}} \cdot\left(\mathrm{a}_{\mathrm{H}^{+}}\right)^{\mathrm{d}}}{\left(\mathrm{a}_{\mathrm{R}}\right)^{\mathrm{a}} \cdot\left(\mathrm{a}_{\mathrm{H}_{2} \mathrm{O}}\right)^{\mathrm{b}}}
$$

is used to relate the cell potential ( $\left.\mathrm{E}^{\prime}\right)$ to the Gibbs free energy of the reaction $\left(\Delta G_{\mathrm{rxn}}\right)$ for each possible redox reaction. $T$ is the temperature, $F$ is the Faraday constant, $R$ is the ideal gas constant, $\mathrm{a}_{P}, \mathrm{a}_{R}, \mathrm{a}_{\mathrm{H}_{2} \mathrm{O}}$, and $\mathrm{a}_{\mathrm{H}^{+}}$are the activity of the reactants, products, water $(=1)$ and $\mathrm{H}^{+}$. The most stable 
species in aqueous solutions can be therefore determined by minimizing $\left(\Delta G_{\mathrm{rxn}}+n F E\right)$ across all possible reactions under certain $\mathrm{pH}$ and applied potential. The energy of all compounds and ions studied in are obtained from the Materials Project ${ }^{5}$ via the Materials Application Programming Interface (API). ${ }^{6,7}$ In this work, the aqueous stability of a material was quantitatively measured by its Gibbs free energy difference $\left(\Delta \mathrm{G}_{\mathrm{pbx}}\right)$ with respect to the stable domains on the Pourbaix diagram under typical ORR/OER operating conditions. 
Table S1: Calculated properties of 53 acid-stable oxides for oxygen electrocatalysis. $\Delta G_{\mathrm{pbx}}^{\mathrm{X}}(\mathrm{X}=$ ORR/OER) (eV/atom) is the maximum aqueous decomposition free energy in the potential range of interest. Band gap (eV) was calculated using the HSE functional. MP-ID is material ID in the Materials Project database. Materials are sorted by their phase stability $\left(E_{\text {hull }}\right)$.

\begin{tabular}{|c|c|c|c|c|c|c|}
\hline Materials & Space Group & $E_{\text {hull }}$ & $\Delta G_{\mathrm{pbx}}$ & Aqueous Decomposition Products & Band Gap & MP-ID \\
\hline $\mathrm{Hg}\left(\mathrm{SbO}_{3}\right)_{2}$ & $\mathrm{P} \overline{3} 1 \mathrm{~m}$ & 0 & $0 / 0$ & $\mathrm{Hg}\left(\mathrm{SbO}_{3}\right)_{2}(\mathrm{~s})(\mathrm{E}=0.6-1.0,1.2-2.0 \mathrm{~V})$ & 1.55 & mp-754065 \\
\hline $\mathrm{Sb}_{2} \mathrm{PbO}_{6}$ & $\mathrm{P} \overline{3} 1 \mathrm{~m}$ & 0 & $0 / 0.103$ & $\begin{array}{l}\mathrm{Sb}_{2} \mathrm{PbO}_{6}(\mathrm{~s})(\mathrm{E}=0.6-1.0,1.2-1.86 \mathrm{~V}) \\
\mathrm{Sb}_{2} \mathrm{O}_{5}(\mathrm{~s})+\mathrm{PbO}_{2}(\mathrm{~s})(\mathrm{E}=1.87-1.95 \mathrm{~V}) \\
\mathrm{Sb}_{3} \mathrm{O}_{8}(\mathrm{~s})+\mathrm{PbO}_{2}(\mathrm{~s})(\mathrm{E}=1.96-2.0 \mathrm{~V})\end{array}$ & 2.81 & mp-20727 \\
\hline $\mathrm{Cd}\left(\mathrm{SbO}_{3}\right)_{2}$ & $\mathrm{P} \overline{3} 1 \mathrm{~m}$ & 0 & $0 / 0$ & $\mathrm{Cd}\left(\mathrm{SbO}_{3}\right)_{2}(\mathrm{~s})(\mathrm{E}=0.6-1.0,1.2-2.0 \mathrm{~V})$ & 2.79 & mp-8922 \\
\hline $\mathrm{BiSbO}_{4}$ & $\mathrm{C} 2 / \mathrm{c}$ & 0 & $0.005 / 0.101$ & $\begin{array}{l}\mathrm{SbO}^{+}+\mathrm{Bi}^{3+}(\mathrm{E}=0.6 \mathrm{~V}) \\
\mathrm{BiSbO}_{4}(\mathrm{~s})(\mathrm{E}=0.61-1.0,1.2-1.9 \mathrm{~V}) \\
\mathrm{Bi}_{2} \mathrm{O}_{5}(\mathrm{~s})+\mathrm{Sb}_{2} \mathrm{O}_{5}(\mathrm{~s})(\mathrm{E}=1.91-1.95 \mathrm{~V}) \\
\mathrm{Bi}_{2} \mathrm{O}_{5}(\mathrm{~s})+\mathrm{Sb}_{3} \mathrm{O}_{8}(\mathrm{~s})(\mathrm{E}=1.96-2.0 \mathrm{~V})\end{array}$ & 3.53 & mp-23018 \\
\hline $\mathrm{LiSb}_{3} \mathrm{O}_{8}$ & $\mathrm{P} 2{ }_{1} / \mathrm{c}$ & 0 & $0.057 / 0.108$ & $\begin{array}{l}\mathrm{Li}^{+}+\mathrm{SbO}^{+}(\mathrm{E}=0.6-0.62 \mathrm{~V}) \\
\mathrm{SbO}_{2}(\mathrm{~s})+\mathrm{Li}^{+}(\mathrm{E}=0.63-0.65 \mathrm{~V}) \\
\mathrm{LiSb}_{3} \mathrm{O}_{8}(\mathrm{~s})(\mathrm{E}=0.66-1.0,1.2-1.92 \mathrm{~V}) \\
\mathrm{LiO}_{3}(\mathrm{~s})+\mathrm{Sb}_{2} \mathrm{O}_{5}(\mathrm{~s})(\mathrm{E}=1.93-1.95 \mathrm{~V}) \\
\mathrm{Sb}_{3} \mathrm{O}_{8}(\mathrm{~s})+\mathrm{LiO}_{3}(\mathrm{~s})(\mathrm{E}=1.96-2.0 \mathrm{~V})\end{array}$ & 2.05 & mp-29892 \\
\hline $\mathrm{KSb}_{5} \mathrm{O}_{13}$ & Ama2 & 0 & $0.060 / 0.069$ & $\begin{array}{l}\mathrm{SbO}^{+}+\mathrm{K}^{+}(\mathrm{E}=0.6-0.62 \mathrm{~V}) \\
\mathrm{K}^{+}+\mathrm{SbO}_{2}(\mathrm{~s})(\mathrm{E}=0.63-0.64 \mathrm{~V}) \\
\mathrm{KSb}_{5} \mathrm{O}_{13}(\mathrm{~s})(\mathrm{E}=0.65-1.0,1.2-1.93 \mathrm{~V}) \\
\mathrm{KO}_{3}(\mathrm{~s})+\mathrm{Sb}_{2} \mathrm{O}_{5}(\mathrm{~s})(\mathrm{E}=1.94-1.95 \mathrm{~V}) \\
\mathrm{KO}_{3}(\mathrm{~s})+\mathrm{Sb}_{3} \mathrm{O}_{8}(\mathrm{~s})(\mathrm{E}=1.96-2.0 \mathrm{~V})\end{array}$ & 1.87 & mp-757115 \\
\hline $\mathrm{Sc}_{2}\left(\mathrm{MoO}_{4}\right)_{3}$ & $\mathrm{Pbcn}$ & 0 & $0 / 0$ & $\mathrm{Sc}_{2}\left(\mathrm{MoO}_{4}\right)_{3}(\mathrm{~s})(\mathrm{E}=0.6-1.0,1.2-2.0 \mathrm{~V})$ & 4.12 & mp-19594 \\
\hline $\mathrm{Ni}\left(\mathrm{SbO}_{3}\right)_{2}$ & $\mathrm{P} 4_{2} / \mathrm{mnm}$ & 0 & $0.004 / 0$ & $\begin{array}{l}\mathrm{SbO}^{+}+\mathrm{Ni}^{2+}(\mathrm{E}=0.6 \mathrm{~V}) \\
\mathrm{Ni}\left(\mathrm{SbO}_{3}\right)_{2}(\mathrm{~s})(\mathrm{E}=0.61-1.0,1.2-2.0 \mathrm{~V})\end{array}$ & 1.33 & $\mathrm{mp}-505271$ \\
\hline $\mathrm{Fe}_{2}\left(\mathrm{MoO}_{4}\right)_{3}$ & Pbcn & 0 & $0.014 / 0.000$ & $\begin{array}{l}\mathrm{Fe}^{2+}+\mathrm{Mo}_{8} \mathrm{O}_{23}(\mathrm{~s})(\mathrm{E}=0.6-0.61 \mathrm{~V}) \\
\mathrm{MoO}_{3}(\mathrm{~s})+\mathrm{Fe}^{2+}(\mathrm{E}=0.62 \mathrm{~V}) \\
\mathrm{Fe}_{2}\left(\mathrm{MoO}_{4}\right)_{3}(\mathrm{~s})(\mathrm{E}=0.63-1.0,1.2-2.0 \mathrm{~V})\end{array}$ & 2.54 & $\mathrm{mp}-31766$ \\
\hline $\mathrm{Fe}_{2}\left(\mathrm{MoO}_{4}\right)_{3}$ & $\mathrm{P} 2{ }_{1} / \mathrm{c}$ & 0 & $0.020 / 0.005$ & $\begin{array}{l}\mathrm{Fe}^{2+}+\mathrm{Mo}_{8} \mathrm{O}_{23}(\mathrm{~s})(\mathrm{E}=0.6-0.61 \mathrm{~V}) \\
\mathrm{Fe}^{2+}+\mathrm{MoO}_{3}(\mathrm{~s})(\mathrm{E}=0.62 \mathrm{~V})\end{array}$ & 2.51 & mp-705435 \\
\hline
\end{tabular}


Table S1 continued from previous page

\begin{tabular}{|c|c|c|c|c|c|c|}
\hline Materials & Space Group & $E_{\text {hull }}$ & $\Delta G_{\mathrm{pbx}}$ & Aqueous Decomposition Products & Band Gap & MP-ID \\
\hline & & & & $\mathrm{Fe}_{2}\left(\mathrm{MoO}_{4}\right)_{3}(\mathrm{~s})(\mathrm{E}=0.63-1.0,1.2-2.0 \mathrm{~V})$ & & \\
\hline $\mathrm{FeSbO}_{4}$ & $\mathrm{Cmmm}$ & 0 & $0.047 / 0.000$ & $\begin{array}{l}\mathrm{Fe}^{2+}+\mathrm{Fe}\left(\mathrm{SbO}_{3}\right)_{2}(\mathrm{~s})(\mathrm{E}=0.6-0.69 \mathrm{~V}) \\
\mathrm{FeSbO}_{4}(\mathrm{~s})(\mathrm{E}=0.7-1.0,1.2-2.0 \mathrm{~V})\end{array}$ & 1.74 & mp-675127 \\
\hline $\mathrm{Sr}\left(\mathrm{SbO}_{3}\right)_{2}$ & $\mathrm{P} \overline{3} 1 \mathrm{~m}$ & 0 & $0.024 /-$ & $\begin{array}{l}\mathrm{Sr}^{2+}+\mathrm{SbO}^{+}(\mathrm{E}=0.6-0.61 \mathrm{~V}) \\
\mathrm{Sr}\left(\mathrm{SbO}_{3}\right)_{2}(\mathrm{~s})(\mathrm{E}=0.62-1.0 \mathrm{~V})\end{array}$ & 3.7 & mp-9126 \\
\hline $\mathrm{Ba}\left(\mathrm{SbO}_{3}\right)_{2}$ & $\mathrm{P} \overline{3} 1 \mathrm{~m}$ & 0 & $0.036 /-$ & $\begin{array}{l}\mathrm{SbO}^{+}+\mathrm{Ba}^{2+}(\mathrm{E}=0.6-0.62 \mathrm{~V}) \\
\mathrm{Ba}^{2+}+\mathrm{SbO}_{2}(\mathrm{~s})(\mathrm{E}=0.63 \mathrm{~V}) \\
\mathrm{Ba}\left(\mathrm{SbO}_{3}\right)_{2}(\mathrm{~s})(\mathrm{E}=0.64-1.0 \mathrm{~V})\end{array}$ & 3.98 & mp-9127 \\
\hline $\mathrm{Ca}\left(\mathrm{SbO}_{3}\right)_{2}$ & $\mathrm{P} \overline{3} 1 \mathrm{~m}$ & 0 & $0.064 /-$ & $\begin{array}{l}\mathrm{Ca}^{2+}+\mathrm{SbO}^{+}(\mathrm{E}=0.6-0.62 \mathrm{~V}) \\
\mathrm{SbO}_{2}(\mathrm{~s})+\mathrm{Ca}^{2+}(\mathrm{E}=0.63-0.67 \mathrm{~V}) \\
\mathrm{Ca}\left(\mathrm{SbO}_{3}\right)_{2}(\mathrm{~s})(\mathrm{E}=0.68-1.0 \mathrm{~V})\end{array}$ & 3.73 & mp-9125 \\
\hline $\mathrm{NaSb}_{5} \mathrm{O}_{13}$ & Ama2 & 0 & $0.073 /-$ & $\begin{array}{l}\mathrm{Na}^{+}+\mathrm{SbO}^{+}(\mathrm{E}=0.6-0.62 \mathrm{~V}) \\
\mathrm{Na}^{+}+\mathrm{SbO}_{2}(\mathrm{~s})(\mathrm{E}=0.63-0.66 \mathrm{~V}) \\
\mathrm{NaSb}_{5} \mathrm{O}_{13}(\mathrm{~s})(\mathrm{E}=0.67-1.0 \mathrm{~V})\end{array}$ & 1.89 & mp-766341 \\
\hline $\mathrm{Ge}_{3} \mathrm{Sb}_{2} \mathrm{O}_{9}$ & $\mathrm{P}_{3} / \mathrm{m}$ & 0 & $0.343 /-$ & $\begin{array}{l}\mathrm{GeO}_{2}(\mathrm{~s})+\mathrm{SbO}^{+}(\mathrm{E}=0.6-0.62 \mathrm{~V}) \\
\mathrm{GeO}_{2}(\mathrm{~s})+\mathrm{SbO}_{2}(\mathrm{~s})(\mathrm{E}=0.63-0.77 \mathrm{~V}) \\
\mathrm{GeO}_{2}(\mathrm{~s})+\mathrm{Sb}_{2} \mathrm{O}_{5}(\mathrm{~s})(\mathrm{E}=0.78-1.0 \mathrm{~V})\end{array}$ & 3.87 & mp-17708 \\
\hline $\mathrm{Ge}_{3}\left(\mathrm{SbO}_{3}\right)_{4}$ & $\mathrm{I} \overline{4} 3 \mathrm{~d}$ & 0 & $0.491 /-$ & $\begin{array}{l}\mathrm{SbO}^{+}+\mathrm{GeO}_{2}(\mathrm{~s})(\mathrm{E}=0.6-0.62 \mathrm{~V}) \\
\mathrm{SbO}_{2}(\mathrm{~s})+\mathrm{GeO}_{2}(\mathrm{~s})(\mathrm{E}=0.63-0.77 \mathrm{~V}) \\
\mathrm{Sb}_{2} \mathrm{O}_{5}(\mathrm{~s})+\mathrm{GeO}_{2}(\mathrm{~s})(\mathrm{E}=0.78-1.0 \mathrm{~V})\end{array}$ & 4.28 & mp-1201722 \\
\hline $\mathrm{Mn}\left(\mathrm{SbO}_{3}\right)_{2}$ & $\mathrm{P} \overline{3} 1 \mathrm{~m}$ & 0 & $0.119 /-$ & $\begin{array}{l}\mathrm{SbO}^{+}+\mathrm{Mn}^{2+}(\mathrm{E}=0.6-0.62 \mathrm{~V}) \\
\mathrm{SbO}_{2}(\mathrm{~s})+\mathrm{Mn}^{2+}(\mathrm{E}=0.63-0.69 \mathrm{~V}) \\
\mathrm{Mn}\left(\mathrm{SbO}_{3}\right)_{2}(\mathrm{~s})(\mathrm{E}=0.7-1.0 \mathrm{~V})\end{array}$ & 1.66 & mp-1078318 \\
\hline $\mathrm{Zn}\left(\mathrm{SbO}_{3}\right)_{2}$ & $\mathrm{P} 4_{2} / \mathrm{mnm}$ & 0 & -10 & $\mathrm{Zn}\left(\mathrm{SbO}_{3}\right)_{2}(\mathrm{~s})(\mathrm{E}=1.2-2.0 \mathrm{~V})$ & 1.82 & mp-3188 \\
\hline $\mathrm{Mg}\left(\mathrm{SbO}_{3}\right)_{2}$ & $\mathrm{P} 4_{2} / \mathrm{mnm}$ & 0 & -10 & $\mathrm{Mg}\left(\mathrm{SbO}_{3}\right)_{2}(\mathrm{~s})(\mathrm{E}=1.2-2.0 \mathrm{~V})$ & 2.28 & $\mathrm{mp}-3653$ \\
\hline $\mathrm{GaSbO}_{4}$ & $\mathrm{Cmmm}$ & 0 & -10 & $\mathrm{GaSbO}_{4}(\mathrm{~s})(\mathrm{E}=1.2-2.0 \mathrm{~V})$ & 1.81 & mp-1224786 \\
\hline $\mathrm{CsSbWO}_{6}$ & Ima2 & 0 & $-/ 0.216$ & $\begin{array}{l}\mathrm{CsSbWO}_{6}(\mathrm{~s})(\mathrm{E}=1.2-1.84 \mathrm{~V}) \\
\mathrm{Sb}_{2} \mathrm{O}_{5}(\mathrm{~s})+\mathrm{WO}_{3}(\mathrm{~s})+\mathrm{Cs}_{2} \mathrm{WO}_{8}(\mathrm{~s})(\mathrm{E}=1.85-1.95 \mathrm{~V}) \\
\mathrm{Sb}_{3} \mathrm{O}_{8}(\mathrm{~s})+\mathrm{WO}_{3}(\mathrm{~s})+\mathrm{Cs}_{2} \mathrm{WO}_{8}(\mathrm{~s})(\mathrm{E}=1.96-2.0 \mathrm{~V})\end{array}$ & 3.32 & mp-1225944 \\
\hline
\end{tabular}


Table S1 continued from previous page

\begin{tabular}{|c|c|c|c|c|c|c|}
\hline Materials & Space Group & $E_{\text {hull }}$ & $\Delta G_{\mathrm{pbx}}$ & Aqueous Decomposition Products & Band Gap & MP-ID \\
\hline $\mathrm{KSb}_{5} \mathrm{O}_{13}$ & $\mathrm{P} 2{ }_{1} / \mathrm{m}$ & 0 & $0.060 / 0.069$ & $\begin{array}{l}\mathrm{SbO}^{+}+\mathrm{K}^{+}(\mathrm{E}=0.6-0.62 \mathrm{~V}) \\
\mathrm{SbO}_{2}(\mathrm{~s})+\mathrm{K}^{+}(\mathrm{E}=0.63-0.64 \mathrm{~V}) \\
\mathrm{KSb}_{5} \mathrm{O}_{13}(\mathrm{~s})(\mathrm{E}=0.65-1.0,1.2-1.93 \mathrm{~V}) \\
\mathrm{KO}_{3}(\mathrm{~s})+\mathrm{Sb}_{2} \mathrm{O}_{5}(\mathrm{~s})(\mathrm{E}=1.94-1.95 \mathrm{~V}) \\
\mathrm{Sb}_{3} \mathrm{O}_{8}(\mathrm{~s})+\mathrm{KO}_{3}(\mathrm{~s})(\mathrm{E}=1.96-2.0 \mathrm{~V})\end{array}$ & 1.85 & mp-1223385 \\
\hline $\mathrm{Fe}_{2}\left(\mathrm{MoO}_{4}\right)_{3}$ & $\mathrm{P} 2_{1}$ & 1 & $0.024 / 0.009$ & $\begin{array}{l}\mathrm{Fe}^{2+}+\mathrm{Mo}_{8} \mathrm{O}_{23}(\mathrm{~s})(\mathrm{E}=0.6-0.61 \mathrm{~V}) \\
\mathrm{MoO}_{3}(\mathrm{~s})+\mathrm{Fe}^{2+}(\mathrm{E}=0.62 \mathrm{~V}) \\
\mathrm{Fe}_{2}\left(\mathrm{MoO}_{4}\right)_{3}(\mathrm{~s})(\mathrm{E}=0.63-1.0,1.2-2.0 \mathrm{~V})\end{array}$ & 2.8 & mp-704851 \\
\hline $\mathrm{FeSbO}_{4}$ & $\mathrm{I} 4_{1} \mathrm{md}$ & 8 & $0.073 / 0.026$ & $\begin{array}{l}\mathrm{Fe}\left(\mathrm{SbO}_{3}\right)_{2}(\mathrm{~s})+\mathrm{Fe}^{2+}(\mathrm{E}=0.6-0.69 \mathrm{~V}) \\
\mathrm{FeSbO}_{4}(\mathrm{~s})(\mathrm{E}=0.7-1.0,1.2-2.0 \mathrm{~V})\end{array}$ & 1.55 & mp-765436 \\
\hline $\mathrm{TiSn}_{9} \mathrm{O}_{20}$ & $\mathrm{C} 2 / \mathrm{m}$ & 13 & $0.041 / 0.041$ & $\mathrm{TiO}_{2}(\mathrm{~s})+\mathrm{SnO}_{2}(\mathrm{~s})(\mathrm{E}=0.6-1.0,1.2-2.0 \mathrm{~V})$ & 2.24 & mp-766163 \\
\hline $\mathrm{FeAg}\left(\mathrm{MoO}_{4}\right)_{2}$ & $\mathrm{P} 2 / \mathrm{c}$ & 17 & $-/ 0.455$ & $\begin{array}{l}\mathrm{Ag}^{+}+\mathrm{Fe}_{2}\left(\mathrm{MoO}_{4}\right)_{3}(\mathrm{~s})+\mathrm{MoO}_{3}(\mathrm{~s})(\mathrm{E}=1.2-1.53 \mathrm{~V}) \\
\mathrm{Fe}_{2}\left(\mathrm{MoO}_{4}\right)_{3}(\mathrm{~s})+\mathrm{MoO}_{3}(\mathrm{~s})+\mathrm{Ag}_{2} \mathrm{O}_{3}(\mathrm{~s})(\mathrm{E}=1.54-2.0 \mathrm{~V})\end{array}$ & 1.43 & mp-1212730 \\
\hline $\mathrm{MoWO}_{6}$ & $\mathrm{Cm}$ & 18 & $0.083 / 0.080$ & $\begin{array}{l}\mathrm{WO}_{3}(\mathrm{~s})+\mathrm{Mo}_{8} \mathrm{O}_{23}(\mathrm{~s})(\mathrm{E}=0.6-0.61 \mathrm{~V}) \\
\mathrm{WO}_{3}(\mathrm{~s})+\mathrm{MoO}_{3}(\mathrm{~s})(\mathrm{E}=0.62-1.0,1.2-2.0 \mathrm{~V})\end{array}$ & 1.37 & mp-1116820 \\
\hline $\mathrm{Ti}\left(\mathrm{Sn}_{2} \mathrm{O}_{5}\right)_{2}$ & $\mathrm{P} \overline{1}$ & 25 & $0.077 / 0.077$ & $\mathrm{SnO}_{2}(\mathrm{~s})+\mathrm{TiO}_{2}(\mathrm{~s})(\mathrm{E}=0.6-1.0,1.2-2.0 \mathrm{~V})$ & 2.71 & mp-766391 \\
\hline $\mathrm{Ti}\left(\mathrm{GeO}_{3}\right)_{2}$ & $\mathrm{C} 2 / \mathrm{c}$ & 27 & $0.083 / 0.083$ & $\mathrm{TiO}_{2}(\mathrm{~s})+\mathrm{GeO}_{2}(\mathrm{~s})(\mathrm{E}=0.6-1.0,1.2-2.0 \mathrm{~V})$ & 4.45 & mp-1042864 \\
\hline $\mathrm{Ti}\left(\mathrm{GeO}_{3}\right)_{2}$ & $\mathrm{P} 2{ }_{1} / \mathrm{c}$ & 28 & $0.084 / 0.084$ & $\mathrm{GeO}_{2}(\mathrm{~s})+\mathrm{TiO}_{2}(\mathrm{~s})(\mathrm{E}=0.6-1.0,1.2-2.0 \mathrm{~V})$ & 4.24 & mp-1043032 \\
\hline $\mathrm{Ti}_{3} \mathrm{Sn}_{7} \mathrm{O}_{20}$ & $\mathrm{Cmmm}$ & 36 & $0.110 / 0.110$ & $\mathrm{TiO}_{2}(\mathrm{~s})+\mathrm{SnO}_{2}(\mathrm{~s})(\mathrm{E}=0.6-1.0,1.2-2.0 \mathrm{~V})$ & 2.87 & mp-765970 \\
\hline $\mathrm{TiSnO}_{4}$ & $\mathrm{Cmmm}$ & 36 & $0.111 / 0.111$ & $\mathrm{TiO}_{2}(\mathrm{~s})+\mathrm{SnO}_{2}(\mathrm{~s})(\mathrm{E}=0.6-1.0,1.2-2.0 \mathrm{~V})$ & 2.69 & mp-1216649 \\
\hline $\mathrm{Ti}_{3}\left(\mathrm{WO}_{6}\right)_{2}$ & $\mathrm{P} 2{ }_{1} / \mathrm{c}$ & 39 & $0.135 / 0.135$ & $\mathrm{TiO}_{2}(\mathrm{~s})+\mathrm{WO}_{3}(\mathrm{~s})(\mathrm{E}=0.6-1.0,1.2-2.0 \mathrm{~V})$ & 2.94 & mp-776763 \\
\hline $\mathrm{Ti}_{2} \mathrm{Sn}_{3} \mathrm{O}_{10}$ & $\mathrm{Cmm} 2$ & 40 & $0.123 / 0.123$ & $\mathrm{TiO}_{2}(\mathrm{~s})+\mathrm{SnO}_{2}(\mathrm{~s})(\mathrm{E}=0.6-1.0,1.2-2.0 \mathrm{~V})$ & 2.88 & mp-757375 \\
\hline $\mathrm{Sn}\left(\mathrm{GeO}_{3}\right)_{2}$ & $\mathrm{C} 2 / \mathrm{c}$ & 42 & $0.127 / 0.127$ & $\mathrm{GeO}_{2}(\mathrm{~s})+\mathrm{SnO}_{2}(\mathrm{~s})(\mathrm{E}=0.6-1.0,1.2-2.0 \mathrm{~V})$ & 3.34 & mp-1042873 \\
\hline $\mathrm{Sn}\left(\mathrm{GeO}_{3}\right)_{2}$ & $\mathrm{P} 2{ }_{1} / \mathrm{c}$ & 43 & $0.130 / 0.130$ & $\mathrm{SnO}_{2}(\mathrm{~s})+\mathrm{GeO}_{2}(\mathrm{~s})(\mathrm{E}=0.6-1.0,1.2-2.0 \mathrm{~V})$ & 3.32 & mp-1043041 \\
\hline $\mathrm{Ti}_{9} \mathrm{SnO}_{20}$ & $\mathrm{C} 2 / \mathrm{m}$ & 43 & $0.131 / 0.131$ & $\mathrm{TiO}_{2}(\mathrm{~s})+\mathrm{SnO}_{2}(\mathrm{~s})(\mathrm{E}=0.6-1.0,1.2-2.0 \mathrm{~V})$ & 2.87 & mp-761148 \\
\hline $\mathrm{TiSnO}_{4}$ & $\mathrm{Cm}$ & 44 & $0.133 / 0.133$ & $\mathrm{SnO}_{2}(\mathrm{~s})+\mathrm{TiO}_{2}(\mathrm{~s})(\mathrm{E}=0.6-1.0,1.2-2.0 \mathrm{~V})$ & 2.94 & mp-753048 \\
\hline
\end{tabular}


Table S1 continued from previous page

\begin{tabular}{|c|c|c|c|c|c|c|}
\hline Materials & Space Group & $E_{\text {hull }}$ & $\Delta G_{\mathrm{pbx}}$ & Aqueous Decomposition Products & Band Gap & MP-ID \\
\hline $\mathrm{FeSbO}_{4}$ & $\mathrm{I} \overline{4} \mathrm{~m} 2$ & 44 & $0.182 / 0.135$ & $\begin{array}{l}\mathrm{Fe}\left(\mathrm{SbO}_{3}\right)_{2}(\mathrm{~s})+\mathrm{Fe}^{2+}(\mathrm{E}=0.6-0.69 \mathrm{~V}) \\
\mathrm{FeSbO}_{4}(\mathrm{~s})(\mathrm{E}=0.7-1.0,1.2-2.0 \mathrm{~V})\end{array}$ & 2.32 & mp-1178209 \\
\hline $\mathrm{Fe}_{2}\left(\mathrm{MoO}_{4}\right)_{3}$ & $\mathrm{P} \overline{1}$ & 45 & $0.175 / 0.160$ & $\begin{array}{l}\mathrm{Fe}^{2+}+\mathrm{Mo}_{8} \mathrm{O}_{23}(\mathrm{~s})(\mathrm{E}=0.6-0.61 \mathrm{~V}) \\
\mathrm{MoO}_{3}(\mathrm{~s})+\mathrm{Fe}^{2+}(\mathrm{E}=0.62 \mathrm{~V}) \\
\mathrm{Fe}_{2}\left(\mathrm{MoO}_{4}\right)_{3}(\mathrm{~s})(\mathrm{E}=0.63-1.0,1.2-2.0 \mathrm{~V})\end{array}$ & 2.52 & mp-1042928 \\
\hline $\mathrm{Ti}_{4} \mathrm{SnO}_{10}$ & $\mathrm{P} \overline{1}$ & 48 & $0.146 / 0.146$ & $\mathrm{SnO}_{2}(\mathrm{~s})+\mathrm{TiO}_{2}(\mathrm{~s})(\mathrm{E}=0.6-1.0,1.2-2.0 \mathrm{~V})$ & 2.69 & mp-766168 \\
\hline $\mathrm{Ti}_{3}\left(\mathrm{SnO}_{5}\right)_{2}$ & $\mathrm{Cmm} 2$ & 49 & $0.149 / 0.149$ & $\mathrm{TiO}_{2}(\mathrm{~s})+\mathrm{SnO}_{2}(\mathrm{~s})(\mathrm{E}=0.6-1.0,1.2-2.0 \mathrm{~V})$ & 2.71 & mp-759737 \\
\hline $\mathrm{Ti}_{7} \mathrm{Sn}_{3} \mathrm{O}_{20}$ & $\mathrm{Cmmm}$ & 53 & $0.161 / 0.161$ & $\mathrm{SnO}_{2}(\mathrm{~s})+\mathrm{TiO}_{2}(\mathrm{~s})(\mathrm{E}=0.6-1.0,1.2-2.0 \mathrm{~V})$ & 2.54 & mp-761118 \\
\hline $\mathrm{TiSnO}_{4}$ & $\mathrm{I} \overline{4} \mathrm{~m} 2$ & 65 & $0.198 / 0.198$ & $\mathrm{SnO}_{2}(\mathrm{~s})+\mathrm{TiO}_{2}(\mathrm{~s})(\mathrm{E}=0.6-1.0,1.2-2.0 \mathrm{~V})$ & 3.05 & mp-773864 \\
\hline $\mathrm{Sn}\left(\mathrm{WO}_{4}\right)_{2}$ & $\mathrm{P} 2 / \mathrm{c}$ & 97 & $0.356 / 0.356$ & $\mathrm{SnO}_{2}(\mathrm{~s})+\mathrm{WO}_{3}(\mathrm{~s})(\mathrm{E}=0.6-1.0,1.2-2.0 \mathrm{~V})$ & 3.19 & mp-1041577 \\
\hline $\mathrm{Ge}_{3}\left(\mathrm{WO}_{6}\right)_{2}$ & $\mathrm{Ia} \overline{3} \mathrm{~d}$ & 100 & $0.343 / 0.343$ & $\mathrm{GeO}_{2}(\mathrm{~s})+\mathrm{WO}_{3}(\mathrm{~s})(\mathrm{E}=0.6-1.0,1.2-2.0 \mathrm{~V})$ & 2.96 & mvc- 4401 \\
\hline $\mathrm{Ti}\left(\mathrm{WO}_{4}\right)_{2}$ & $\mathrm{P} 2 / \mathrm{c}$ & 110 & $0.406 / 0.406$ & $\mathrm{TiO}_{2}(\mathrm{~s})+\mathrm{WO}_{3}(\mathrm{~s})(\mathrm{E}=0.6-1.0,1.2-2.0 \mathrm{~V})$ & 2.89 & mp-1041486 \\
\hline $\mathrm{Zn}\left(\mathrm{SbO}_{3}\right)_{2}$ & $\operatorname{Pmn} 2_{1}$ & 115 & -10.346 & $\mathrm{Zn}\left(\mathrm{SbO}_{3}\right)_{2}(\mathrm{~s})(\mathrm{E}=1.2-2.0 \mathrm{~V})$ & 2.04 & mp-1045304 \\
\hline $\mathrm{TiFeSbO}_{6}$ & $\mathrm{P}_{2}{ }_{2} \mathrm{~nm}$ & 119 & $0.389 / 0.358$ & $\begin{array}{l}\mathrm{Fe}^{2+}+\mathrm{TiO}_{2}(\mathrm{~s})+\mathrm{Fe}\left(\mathrm{SbO}_{3}\right)_{2}(\mathrm{~s})(\mathrm{E}=0.6-0.69 \mathrm{~V}) \\
\mathrm{FeSbO}_{4}(\mathrm{~s})+\mathrm{TiO}_{2}(\mathrm{~s})(\mathrm{E}=0.7-2.0 \mathrm{~V})\end{array}$ & 2.13 & mp-1216868 \\
\hline $\mathrm{Ge}_{3}\left(\mathrm{MoO}_{6}\right)_{2}$ & $\mathrm{Ia} \overline{3} \mathrm{~d}$ & 132 & $0.456 / 0.454$ & $\begin{array}{l}\mathrm{Mo}_{8} \mathrm{O}_{23}(\mathrm{~s})+\mathrm{GeO}_{2}(\mathrm{~s})(\mathrm{E}=0.6-0.61 \mathrm{~V}) \\
\mathrm{GeO}_{2}(\mathrm{~s})+\mathrm{MoO}_{3}(\mathrm{~s})(\mathrm{E}=0.62-1.0,1.2-2.0 \mathrm{~V})\end{array}$ & 2.44 & mvc-4408 \\
\hline $\mathrm{Mg}\left(\mathrm{SbO}_{3}\right)_{2}$ & $\operatorname{Pmn} 2_{1}$ & 142 & -10.426 & $\mathrm{Mg}\left(\mathrm{SbO}_{3}\right)_{2}(\mathrm{~s})(\mathrm{E}=1.2-2.0 \mathrm{~V})$ & 2.31 & mp-1048765 \\
\hline $\mathrm{Sr}\left(\mathrm{SbO}_{3}\right)_{2}$ & $\mathrm{C} 2 / \mathrm{c}$ & 145 & $0.460 /-$ & $\begin{array}{l}\mathrm{Sr}^{2+}+\mathrm{SbO}^{+}(\mathrm{E}=0.6-0.61 \mathrm{~V}) \\
\mathrm{Sr}\left(\mathrm{SbO}_{3}\right)_{2}(\mathrm{~s})(\mathrm{E}=0.62-1.0 \mathrm{~V})\end{array}$ & 2.5 & mp-675680 \\
\hline
\end{tabular}




\section{References}

(1) Kresse, G.; Furthmüller, J. Efficient iterative schemes for $<\mathrm{i}>$ ab initio $</ \mathbf{i}>$ total-energy calculations using a plane-wave basis set. Physical Review B 1996, 54, 11169-11186.

(2) Blöchl, P. E. Projector augmented-wave method. Physical Review B 1994, 50, 17953-17979.

(3) Heyd, J.; Scuseria, G. E.; Ernzerhof, M. Hybrid functionals based on a screened Coulomb potential. Journal of Chemical Physics 2003, 118, 8207-8215.

(4) Heyd, J.; Scuseria, G. E.; Ernzerhof, M. Erratum: Hybrid functionals based on a screened Coulomb potential (Journal of Chemical Physics (2003) 118 (8207)). Journal of Chemical Physics 2006, 124, 219906.

(5) Jain, A.; Ong, S. P.; Hautier, G.; Chen, W.; Richards, W. D.; Dacek, S.; Cholia, S.; Gunter, D.; Skinner, D.; Ceder, G.; Persson, K. A. Commentary: The materials project: A materials genome approach to accelerating materials innovation. APL Materials 2013, 1, 011002.

(6) Ong, S. P.; Cholia, S.; Jain, A.; Brafman, M.; Gunter, D.; Ceder, G.; Persson, K. A. The Materials Application Programming Interface (API): A simple, flexible and efficient API for materials data based on REpresentational State Transfer (REST) principles. Computational Materials Science 2015, 97, 209-215.

(7) Ong, S. P.; Richards, W. D.; Jain, A.; Hautier, G.; Kocher, M.; Cholia, S.; Gunter, D.; Chevrier, V. L.; Persson, K. A.; Ceder, G. Python Materials Genomics (pymatgen): A robust, open-source python library for materials analysis. Computational Materials Science 2013, 68, 314-319.

(8) Persson, K. A.; Waldwick, B.; Lazic, P.; Ceder, G. Prediction of solid-aqueous equilibria: Scheme to combine first-principles calculations of solids with experimental aqueous states. Physical Review B 2012, 85, 235438. 
(9) Singh, A. K.; Zhou, L.; Shinde, A.; Suram, S. K.; Montoya, J. H.; Winston, D.; Gregoire, J. M.; Persson, K. A. Electrochemical Stability of Metastable Materials. Chemistry of Materials 2017, 29, 10159-10167.

(10) Patel, A.; Nørskov, J. K.; Persson, K. A.; Montoya, J. H. Efficient Pourbaix diagrams of many-element compounds. 2019, 1-11. 